\title{
Screening for Compounds with Aromatase Inhibiting Activities from Atractylodes macrocephala Koidz
}

\author{
Hai Jiang ${ }^{1}$, Jing Shi ${ }^{2}$ * and Yuanyuan $\mathrm{Li}^{2}$
}

1 Department of Urology, First Affiliated Hospital, School of Medicine, Zhejiang University, No.79 Qingchun Rd., Hangzhou 310003, China; E-Mail: jhai123@yahoo.com.cn

2 Department of Pharmacy, Zhejiang Medical College, No.481 Binwen Rd., Hangzhou 310053, China

* Author to whom correspondence should be addressed; E-Mail: shij136@hotmail.com; Tel.: +86-571-87692881; Fax: +86-571-87692832.

Received: 31 March 2011; in revised form: 11 April 2011 / Accepted: 12 April 2011 / Published: 14 April 2011

\begin{abstract}
Ten compounds were isolated from the dichloromethane extract of Atractylodes macrocephala and their aromatase inhibiting activities were tested using an in vitro fluorescent-based aromatase assay. The results indicated that atractylenolide I (1), atractylenolide II (2) and atractylenolide III (3) had inhibition ratios of $94.56 \pm 0.70 \%$, $90.93 \pm 1.41 \%$ and $86.31 \pm 8.46 \%$, respectively, at a concentration of $10 \mu \mathrm{M}$. We conclude from our results that atractylenolide and its derivates may serve as potential aromatase inhibitors (AIs) and thus merit continued study in the future.
\end{abstract}

Keywords: aromatase; Atractylodes macrocephala; atractylenolide

\section{Introduction}

Breast cancer is one of the most commonly diagnosed types of cancer in women. In the United States, breast cancer is the second leading cause of death from cancer in women. About two-thirds premenopausal and three-quarters of postmenopausal breast cancer patients have estrogen-dependent cancer, which means estrogens play a critical role in stimulating breast cancer cell proliferation [1]. Therefore, antagonizing the action of estrogen can depress the development of breast cancer. According to the previous studies, there are two main approaches to control the pathological activity of 
estrogens [2]. The first is targeting the estrogen receptor directly, which results in the discovery of estrogen receptor antagonists tamoxifen and raloxifene $[3,4]$. The second is inhibiting aromatase (also called CYP19), a key enzyme that belongs to one of the subfamilies of cytochrome P450s and catalyzes the conversion of androgens to estrogens by altering the steroid enone A-ring to aromatic phenolic ring (Figure 1) [5]. From this point of view, aromatase is a seemly molecular target in the treatment of estrogen receptor sensitive breast cancer. Many aromatase inhibitors (AIs) were developed for the treatment of breast cancer, such as aminoglutethimide (AG), the first AI used in clinic and was ultimately withdrew due to its high toxicity and low selectivity [6]. Other effective AIs were formestane, exemestane, anastrozole and letrozole, which are remarkably potent and sufficiently selective drugs. AIs can be both steroidal and non-steroidal compounds [7].

The rhizomes of Atractylodes macrocephala Koidz ("Baizhu" in Chinese) is one of the most popular Traditional Chinese Medicines (TCMs), which has a long history of use for the treatment of splenic asthenia, anorexia, oedema, excessive perspiration and abnormal fetal movement. Previous chemical investigations on the rhizomes of $A$. macrocephala demonstrated that the main active constituents in them were sesquiterpenes and acetylenic compounds [8-10], which have been proven to possess anti-tumor and anti-inflammatory activities $[11,12]$. In the present study, ten compounds were isolated from the dichloromethane extract of A. macrocephala, which presented potent aromatase inhibiting activities.

Figure 1. Estrone and estradiol biosynthesis mediated by aromatase $(\mathrm{R}=\mathrm{O}, \mathrm{OH})$.

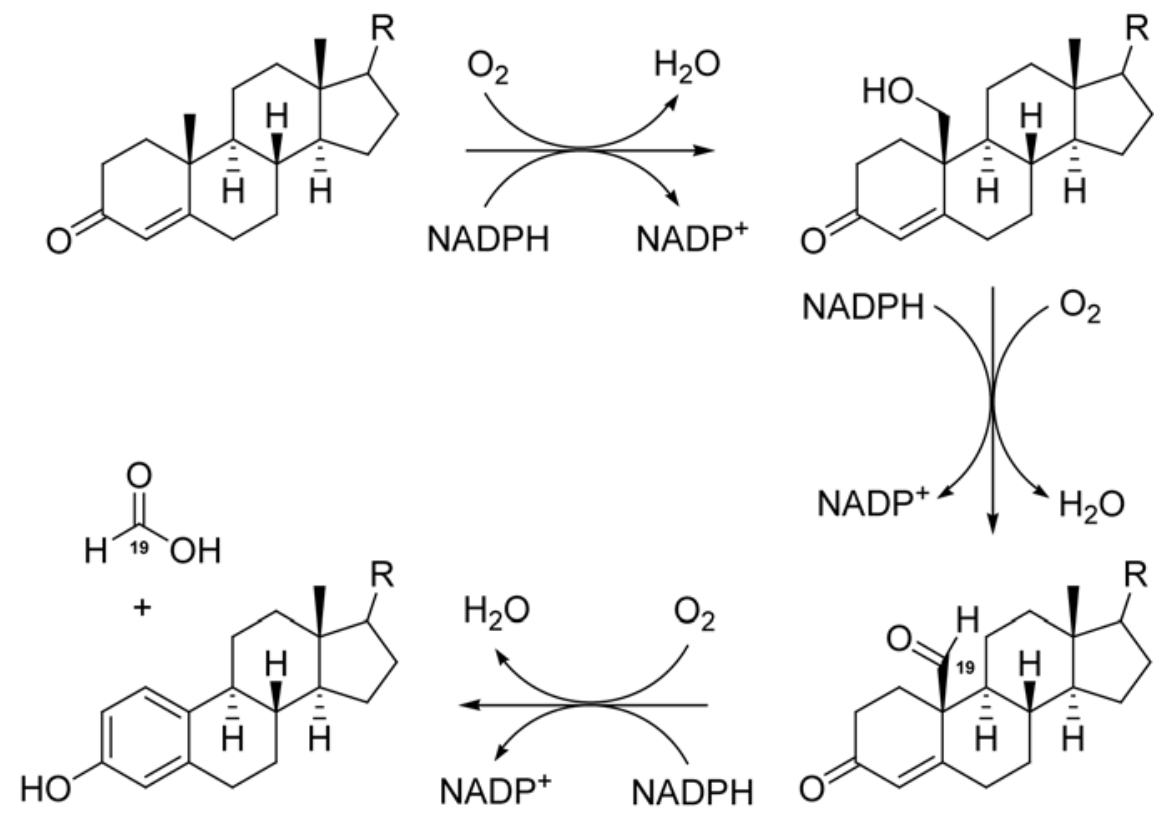

\section{Results and Discussion}

The dichloromethane extract of A. macrocephala yielded ten known compounds (Figure 2): atractylenolide I (1), atractylenolide II (2), atractylenolide III (3), biatractylenolide (4), taraxeryl acetate (5), $\beta$-sitosterol (6), stigmasterol (7), juniper camphor (8), atractyloside A (9) and eudesm4(15),7-diene-9 $\alpha, 11$-diol (10). Their structures were elucidated on the basis of the results of NMR, MS and IR spectroscopic analysis and compared with the precious references [11-14]. 
Figure 2. Chemical structures of compounds 1-11.

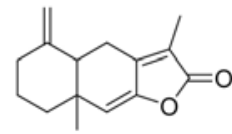

atractylenolide I (1)

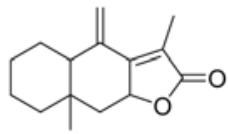

atractylenolide II (2)

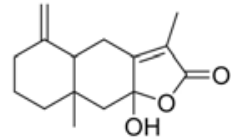

atractylenolide III (3)

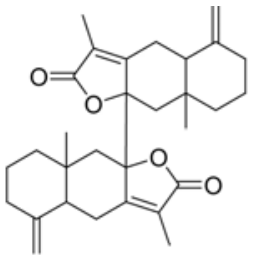

biatractylenolide (4)

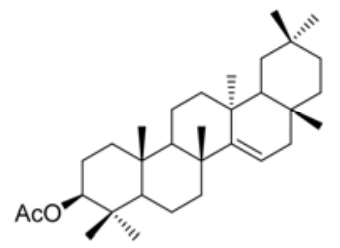

taraxeryl acetate (5)

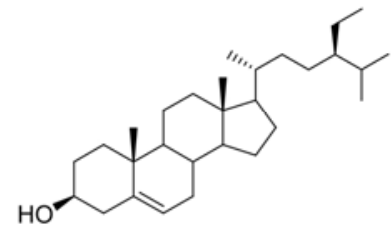

$\beta$-sitosterol (6)

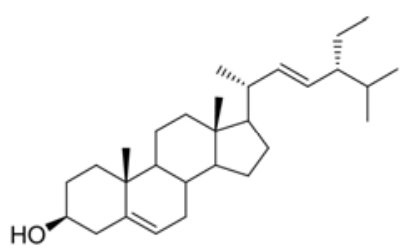

stigmasterol (7)

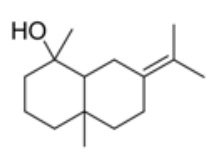

juniper camphor (8)

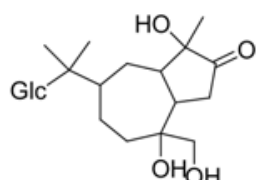

atractyloside A (9)<smiles>C=C1CCCC2C1C(C(C)(C)O)=CC(O)C2C</smiles>

eudesm-4(15),7-diene-9a,11-diol (10)

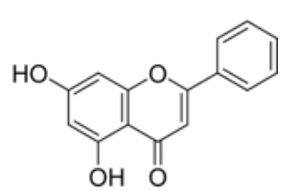

chrysin (11)

Since the dichloromethane extract of $A$. macrocephala showed aromatase inhibiting activity (the inhibitory rate was $78.17 \pm 9.76 \%$ ), we further tested the aromatase inhibiting activities of compounds 1-10 isolated from the extract. The results (Figure 3) indicated that all the compounds presented aromatase inhibiting activities. The aromatase inhibiting activities of compounds 1-3 were the most potent (the inhibitory rates were $94.56 \pm 0.70 \%, 90.93 \pm 1.41 \%$ and $86.31 \pm 8.46 \%$, respectively). Chrysin was tested as the positive control and its aromatase inhibitory rate was $93.30 \pm 5.65 \%$. Comparing the structures of ten isolated compounds, compounds 1-3 shared a common structural characteristic, which might play a critical role in their aromatase inhibiting activities. Therefore, it was suggested that atractylenolide and its derivates might serve as potential AIs and certainly merited continued and comparative study for the future.

Figure 3. Aromatase inhibitory rates of the dichloromethane extract of A. macrocephala, compounds 1-10 and chrysin.

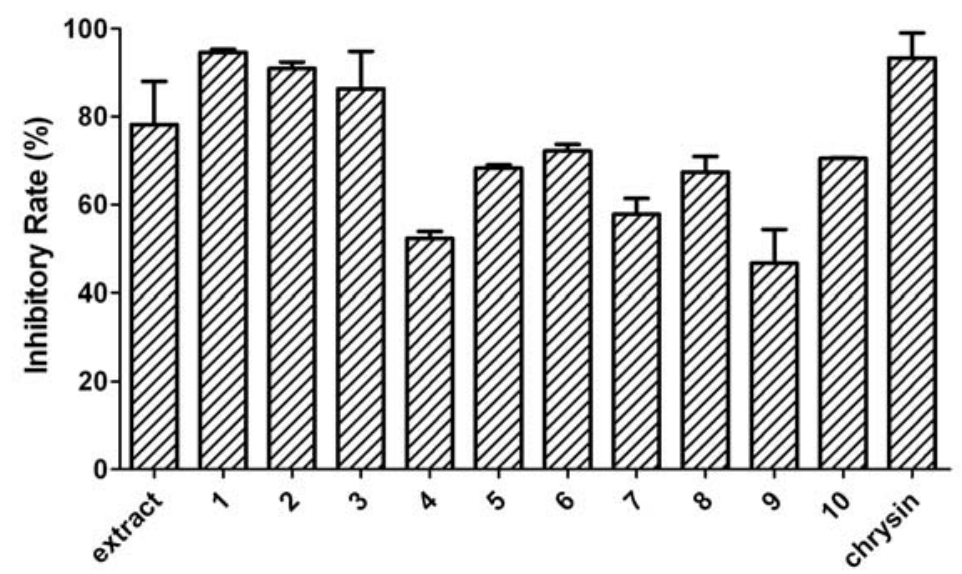




\section{Experimental}

\subsection{General}

IR spectra were measured on a JASCO FTIR4100 spectrophotometer. ${ }^{1} \mathrm{H}$ - and ${ }^{13} \mathrm{C}$-NMR spectra were recorded on a Bruker Ultrashield Plus $500 \mathrm{MHz}$ spectrometer. The mass spectra were recorded using an LCQ Deca XP ${ }^{\text {plus }}$ ESI ion trap mass spectrometer (Thermo Finnigan, USA). Silica gel (300-400 mesh, Qingdao Chemical Group, China) was employed for column chromatography. The preparative HPLC experiments were performed on an Agilent 1200 HPLC system (Agilent Technologies, Germany) using Zorbax SB-C 18 column $(21.2 \mathrm{~mm} \times 250 \mathrm{~mm}, 10 \mu \mathrm{m})$. Aromatase (Human CYP19 + P450 Reductase supersomes ${ }^{\mathrm{TM}}$ ) was purchased from BD Biosciences (Woburn, MA, USA). Chrysin was purchased from National Institute for the Control of Pharmaceutical and Biological Products (Beijing, China).

\subsection{Plant materials}

The rhizomes of $A$. macrocephala were purchased from the traditional Chinese medicine market at Hangzhou City, Zhejiang Province, P.R. China, in June 2008 and identified by the authors. A voucher specimen (No. AM080603) was deposited in the herbarium of Department of Pharmacy, Zhejiang Medical College.

\subsection{Extraction and isolation}

The dried rhizomes of $A$. macrocephala $(10 \mathrm{~kg})$ were extracted with $95 \%$ ethanol $(1 \mathrm{~L} \times 4)$ to give a crude ethanol extract. The residue was dissolved in $\mathrm{H}_{2} \mathrm{O}(1 \mathrm{~L})$ and then extracted successively with petroleum ether $(1 \mathrm{~L} \times 3)$ and dichloromethane $(1 \mathrm{~L} \times 3)$. The dichloromethane part was subjected to column chromatography (silica gel), eluted with petroleum ether/ethyl acetate [100:5, 100:10, 100:20, 100:50, 100:100 (v/v)] and dichloromethane/methanol [100:10, 100:20 (v/v)] to afford seven fractions (Fr. A-G). Fr. A was further separated by preparative HPLC to yield compounds 1, 2, 4, 5 and 8. Fr. B was further separated by preparative HPLC to yield compounds $\mathbf{3}$ and $\mathbf{6}$. Fr. C was further separated by preparative HPLC to yield compound 7. Fr. D was further separated by preparative HPLC to yield compound 10. Fr. G was further separated by preparative HPLC to yield compound $\mathbf{9}$.

\subsection{Aromatase inhibition assay}

Aromatase inhibition was evaluated by measuring the fluorescent intensity of fluorescein, the hydrolysis product of dibenzylfluorescein (DBF), by aromatase as described previously [15]. Compounds 1-10 and the dichloromethane extract of $A$. macrocephala were tested for their aromatase inhibiting activities. Briefly, the test sample $(10 \mu \mathrm{L})$ was preincubated with a NADPH regenerating system (2.6 mM NADP ${ }^{+}, \quad 7.6 \mathrm{mM}$ glucose-6-phosphate, $0.8 \mathrm{U} / \mathrm{mL}$ glucose-6-phosphate dehydrogenase, $13.9 \mathrm{mM} \mathrm{MgCl}_{2}$, and $1 \mathrm{mg} / \mathrm{mL}$ albumin in $90 \mu \mathrm{L}$ of $50 \mathrm{mM}$ potassium phosphate, $\mathrm{pH}$ 7.4) for $10 \mathrm{~min}$ at $37^{\circ} \mathrm{C}$. The final concentration of extract and each compound were $100 \mu \mathrm{g} / \mathrm{mL}$ and $10 \mu \mathrm{M}$, respectively. Then the enzyme and substrate mixture $(0.8 \mu \mathrm{L} \mathrm{DBF}, 40 \mathrm{pmol} / \mathrm{mL}$ of aromatase, and $4 \mathrm{mg} / \mathrm{mL}$ albumin in $100 \mu \mathrm{L}$ of $50 \mathrm{mM}$ potassium phosphate, $\mathrm{pH}$ 7.4) were added to 
incubate for an additional $30 \mathrm{~min}$ at $37^{\circ} \mathrm{C}$. Finally, the fluorescence of reaction solvent was measured using infinite F200 scanning fluorescence plate reader (Tecan, Mannedorf, Switzerland) with excitation at $485 \mathrm{~nm}$ and emission at $535 \mathrm{~nm}$. Chrysin was tested as positive control. Experiments were carried out three times on separate occasions.

\section{Conclusions}

In the present study, ten compounds were isolated from the dichloromethane extract of $A$. macrocephala and their aromatase inhibiting activities were tested using an in vitro fluorescence-based aromatase assay. Three compounds - atractylenolide I (1), atractylenolide II (2) and atractylenolide III (3) - showed potent aromatase inhibiting activities, with aromatase inhibition ratios of $94.56 \pm 0.70 \%$, $90.93 \pm 1.41 \%$ and $86.31 \pm 8.46 \%$, respectively. In summary, atractylenolide and its derivates might serve as potential AIs and merit further study in the future.

\section{Acknowledgements}

This research work is financially supported by Foundation of Zhejiang Administration of Traditional Chinese Medicine (No. 2009CA045), Foundation of Zhejiang Educational Committee (No. Y200805085) and Foundation of Zhejiang Medical College (No. 2008XZA01).

\section{References}

1. Brueggemeier, R.W.; Richards, J.A.; Joomprabutra, S.; Bhat, A.S.; Whetstone, J.L.J. Molecular pharmacology of aromatase and its regulation by endogenous and exogenous agents. J. Steroid Biochem. Mol. Biol. 2001, 79, 75-84.

2. Leonetti, F.; Favia, A.; Rao, A.; Aliano, R.; Paluszcak, A.; Hartmann, R.W.; Carotti, A. Design, synthesis, and 3D QSAR of novel potent and selective aromatase inhibitors. J. Med. Chem. 2004, 47, 6792-6803.

3. Lonard, D.M.; Smith, C.L. Molecular perspectives on selective estrogen receptor modulators (SERMs): Progress in understanding their tissue-specific agonist and antagonist actions. Steroids 2002, 67, 15-24.

4. Park, W.C.; Jordan, V.C. Selective estrogen receptor modulators (SERMS) and their roles in breast cancer prevention. Trends Mol. Med. 2002, 8, 82-88.

5. Ghosh, D.; Griswold, J.; Erman, M.; Pangborn, W. Structural basis for androgen specificity and oestrogen synthesis in human aromatase. Nature 2009, 457, 219-223.

6. Santen, R.J.; Samojlik, E.; Lipton, A.; Harvey, H.; Ruby, E.B.; Wells, S.A.; Kendall, J. Kinetic, hormonal and clinical studies with aminoglutethimide in breast-cancer. Cancer 1977, 39, 2948-2958.

7. O'Reilly, J.M.; Brueggemeier, R.W. 7 $\alpha$-Arylaliphatic androsta-1,4-diene-3,17-diones as enzymeactivated irreversible inhibitors of aromatase. J. Steroid Biochem. Mol. Biol. 1996, 159, 93-102.

8. Chen, Z.L. The acetylenes from Atractylodes macrocephala. Planta Med. 1987, 53, 493-494.

9. Endo, K.; Taguchi, T.; Taguchi, F.; Hikino, H.; Yamahara, J.; Fujimura, H. Anti-inflammatory principles of Atractylodes rhizomes. Chem. Pharm. Bull. 1979, 27, 2954-2958. 
10. Huang, B.S.; Sun, J.S.; Chen, Z.L. Isolation and identification of atractylenolide VI from Atractylodes macrocephala Koidz. Acta Bot. Sin. 1992, 34, 614-617.

11. Wang, H.X.; Liu, C.M.; Liu, Q.; Gao, K. Three types of sesquiterpenes from rhizomes of Atractylodes lancea. Phytochemistry 2008, 69, 2088-2094.

12. Li, C.Q.; He, L.C.; Dong, H.Y.; Jin, J.Q. Screening for the anti-inflammatory activity of fractions and compounds from Atractylodes macrocephala koidz. J. Ethnopharmacol. 2007, 114, 212-217.

13. Dong, H.Y.; He, L.C.; Huang, M.; Dong, Y.L. Anti-inflammatory components isolated from Atractylodes macrocephala Koidz. Nat. Prod. Res. 2008, 22, 1418-1427.

14. Peng, W.; Han, T.; Wang, Y.; Xin, W.B.; Zheng, C.J.; Qin, L.P. Chemical constituents of the aerial part of Atractylodes macrocephala. Chem. Nat. Compd. 2011, 46, 959-960.

15. Endringer, D.C.; Guimaraes, K.G.; Kondratyuk, T.P.; Pezzuto, J.M.; Braga, F.C. Selective inhibition of aromatase by a dihydroisocoumarin from Xyris pterygoblephara. J. Nat. Prod. 2008, 71, 1082-1084.

Sample Availability: Samples of the compounds 1-10 are available from the authors.

(C) 2011 by the authors; licensee MDPI, Basel, Switzerland. This article is an open access article distributed under the terms and conditions of the Creative Commons Attribution license (http://creativecommons.org/licenses/by/3.0/). 